\title{
A Randomized Placebo-Controlled Pilot Trial of Omega-3 Fatty Acids and Alpha Lipoic Acid in Alzheimer's Disease
}

\author{
Lynne Shinto ${ }^{\mathrm{a}, *}$, Joseph Quinn ${ }^{\mathrm{a}, \mathrm{b}}$, Thomas Montine ${ }^{\mathrm{c}}$, Hiroko H. Dodge ${ }^{\mathrm{a}}$, William Woodward ${ }^{\mathrm{a}}$, \\ Sara Baldauf-Wagner ${ }^{\mathrm{a}}$, Dana Waichunas ${ }^{\mathrm{a}}$, Lauren Bumgarner ${ }^{\mathrm{a}}$, Dennis Bourdette ${ }^{\mathrm{a}, \mathrm{b}}$, Lisa Silbert ${ }^{\mathrm{a}, \mathrm{b}}$ \\ and Jeffrey Kaye $\mathrm{e}^{\mathrm{a}, \mathrm{b}}$ \\ ${ }^{a}$ Department of Neurology, Oregon Health \& Science University, Portland, OR, USA \\ ${ }^{\mathrm{b}}$ Department of Veterans Affairs Medical Center, Portland, OR, USA \\ ${ }^{\mathrm{c}}$ Department of Pathology, University of Washington, Seattle, WA, USA
}

Accepted 24 June 2013

\begin{abstract}
Oxidative stress, inflammation, and increased cholesterol levels are all mechanisms that have been associated with Alzheimer's disease (AD) pathology. Several epidemiologic studies have reported a decreased risk of AD with fish consumption. This pilot study was designed to evaluate the effects of supplementation with omega- 3 fatty acids alone $(\omega-3)$ or omega- 3 plus alpha lipoic acid $(\omega-3+\mathrm{LA})$ compared to placebo on oxidative stress biomarkers in AD. The primary outcome measure was peripheral F2-isoprostane levels (oxidative stress measure). Secondary outcome measures included performance on: Mini-Mental State Examination (MMSE), Activities of Daily Living/Instrumental Activities of Daily Living (ADL/IADL), and Alzheimer Disease Assessment Scale-cognitive subscale (ADAS-cog). Thirty-nine AD subjects were randomized to one of three groups: 1) placebo, 2) $\omega-3$, or 3) $\omega-3+$ LA for a treatment duration of 12 months. Eighty seven percent (34/39) of the subjects completed the 12-month intervention. There was no difference between groups at 12 months in peripheral F2-isoprostane levels $(p=0.83)$. The $\omega-3+\mathrm{LA}$ and $\omega-3$ were not significantly different than the placebo group in ADAS-cog $(p=0.98, p=0.86)$ and in ADL $(p=0.15, p=0.82)$. Compared to placebo, the $\omega-3+$ LA showed less decline in MMSE $(p<0.01)$ and IADL $(p=0.01)$ and the $\omega-3$ group showed less decline in IADL $(p<0.01)$. The combination of $\omega-3+$ LA slowed cognitive and functional decline in AD over 12 months. Because the results were generated from a small sample size, further evaluation of the combination of omega-3 fatty acids plus alpha-lipoic acid as a potential treatment in AD is warranted.
\end{abstract}

Keywords: Alpha-lipoic acid, Alzheimer's disease, clinical trial, omega-3 fatty acids

\section{INTRODUCTION}

Although significant progress has been made in both understanding some of the mechanisms of Alzheimer's disease $(\mathrm{AD})$ pathology and developing therapeutic agents, these efforts have had no impact on decreasing disease prevalence and have had limited effects on improving the clinical course of $\mathrm{AD}$ [1]. The exponen-

\footnotetext{
*Correspondence to: Lynne Shinto, ND, MPH, Department of Neurology, Oregon Health \& Science University, 3181 SW Sam Jackson Pk. Rd., CR120, Portland, OR 97239, USA. Tel.: +1 503 494 5035; Fax: +1 503494 0966; E-mail: shintol@ohsu.edu.
}

tial rise in the prevalence, incidence, and cost of care for $\mathrm{AD}$ make finding therapeutic agents that can delay disease progression an urgent public health concern.

Omega-3 fatty acids ( $\omega-3)$ are a family of polyunsaturated fatty acids (PUFAs) that contain a common carbon-carbon double-bond at the third carbon from the terminal methyl end of the molecule. The parent $\omega-3$ is linolenic acid, it is an 'essential fatty acid' and cannot be synthesize in humans, and therefore it must be supplied in the diet. Eicosapentaenoic acid (EPA) and docosahexaenoic acid (DHA) are two types of $\omega-3$ that are synthesized from linolenic acid through 
a series of enzymatic steps [2]. High concentrations of both EPA and DHA can be found in cold water fish and fish oils. DHA is the major PUFA present in the phospholipid fractions of the brain [3] and EPA is a potent anti-inflammatory agent and can displace arachidonic acid in immune cell membranes to affect T-cell signaling and antigen presentation [4]. A number of epidemiologic studies have observed a consistent association of a decreased risk for AD and greater fish consumption [5-7].

Alpha lipoic acid (LA) is a naturally occurring dithiol compound that is synthesized in the mitochondria. Dietary sources of LA include meat, heart, kidneys, liver, and small amounts from fruits and vegetables. Only small amounts of LA can be obtained from the diet and therefore, most information on clinical effects come from studies that supplement LA [8]. It is a powerful antioxidant and can recycle other antioxidants such as vitamin C, vitamin E, and glutathione [9]. It has anti-inflammatory properties and has been shown to reduce NF- $\kappa \mathrm{B}$ activity in vitro in cells stimulated with TNF- $\alpha$ in a dose dependent manner [10].

The primary aim of this study was to evaluate the effects of $\omega$-3 (fish oil concentrate) alone and in combination with $\mathrm{LA}$ on oxidative stress in $\mathrm{AD}$. The primary outcome measure was peripheral F2-isoprostane levels which is a measure of lipid oxidation. LA was added to enhance the potential treatment effects of $\omega-3$, as it could mitigate oxidation of the fish oil supplement, and might also have direct antioxidant effects in vivo. Secondary outcomes included measures of cognition [Alzheimer Disease Assessment Scale-cognitive subscale (ADAS-cog), Mini-Mental State Examination (MMSE)] and a measure of functional ability [Activities of Daily Living/Instrumental Activities of Daily Living (ADL/IADL)]. We hypothesized that each treatment would have a greater effect compared to placebo on lipid oxidation and clinical outcomes, with the combination therapy showing the greatest effect on all outcomes.

\section{MATERIALS AND METHODS}

\section{Participants}

All participants and study partners provided written informed consent before entering the study. Inclusion criteria were age 55 years or older, diagnosis of probable AD (NINCDS/ADRDA criteria), MMSE score 15-26, Clinical Dementia Rating Scale 0.5 to 1.0 , not depressed (Center for Epidemiological Studies of Depression Score <4.0), general health status that would not interfere with patient's ability to participate and complete the study, and caregiver/informant able to accompany participant to study visits. Exclusion criteria were non-AD dementia, residence in a long-term care facility at screening visit, history of clinically significant stroke, health conditions such as cancer (prostate cancer gleason grade $<3$ and non-metastatic cancers were acceptable), liver disease, history of ventricular fibrillation or ventricular tachycardia, major psychiatric disorder, major central nervous system diseases (e.g., brain tumor, seizure disorder), taking lipid lowering medication, hyperlipidemic (triglycerides $>500 \mathrm{mg} / \mathrm{dl}$, LDL $>160 \mathrm{mg} / \mathrm{dl}$, Total cholesterol $>240 \mathrm{mg} / \mathrm{dl}$ ), fish oil or cod liver oil supplementation within 30 days of enrollment, greater than one 6 ounce serving per week of fish or seafood within 30 days of enrollment, lipoic acid supplementation within 30 days of enrollment, taking systemic corticosteroids, neuroleptics, antiparkinsonian agents, and narcotic analgesics. The following medications and supplements were allowed if stable for four months prior to study enrollment, acetylcholinesterase inhibitors, memantine, vitamin E, and ginkgo biloba. This study was approved and monitored by Oregon Health \& Science University's Institutional Review Board and registered at ClincalTrials.gov, NCT00090402.

\section{Study design and randomization}

This study was designed as a 3-arm, parallel group, randomized, double-blind, placebo-controlled pilot clinical trial to test the safety and efficacy of $\omega-3$ and $\omega-3+\mathrm{LA}$ with a 12 -month treatment period. The study sample size was powered to enroll the number needed to see a difference in F2-isoprostane levels between placebo and either treatment arm. After meeting screening eligibility criteria, 39 subjects were randomized to one of three groups (13/group): 1) placebo; 2) $\omega-3$ (fish oil concentrate containing a daily dose of $675 \mathrm{mg}$ DHA and $975 \mathrm{mg}$ EPA; or 3) $\omega$-3 (fish oil concentrate containing a daily dose of $675 \mathrm{mg}$ DHA and $975 \mathrm{mg}$ EPA) plus LA (600 mg/day). Participants were randomized by a computer-generated scheme that was stratified by smoking status (current smoker versus nonsmoker) as this would have the greatest impact on the primary outcome [11].

\section{Outcome measures}

The primary outcome measure was a change in urine F2-isoprostane levels (adjusted for creatinine) from baseline to 12-months. Secondary outcome measures 
included a change from baseline to 12-months on the following: ADAS-cog, MMSE, ADL, IADL.

\section{Study drugs}

$\omega-3$ only group: $\omega-3$ was given in the form of fish oil concentrate in the triglyceride form at 3 grams/day, containing a daily dose of $675 \mathrm{mg}$ DHA and $975 \mathrm{mg}$ EPA and flavored with lemon to mask the fishy taste. Each participant was instructed to take 2 capsules in the morning with food and 1 capsule in the afternoon with food. These participants also took 1 placebo LA tablet in the morning. $\omega-3+$ LA group: LA was given in the racemic form at $600 \mathrm{mg} /$ day in one tablet. Each participant randomized to the $\omega-3+$ LA group took 1 LA tablet and 2 fish oil capsules in the morning with a meal and 1 fish oil capsule in the afternoon with a meal. Placebo group: The placebo LA contained no LA and the following excipients, lactose, hypromellose, silicon dioxide, microcristalline cellulose, polyethylene glycol, povidone, corn starch, talc, and magnesium stearate. The placebo oil contained soybean oil that contained 5\% fish oil and lemon flavor to match the fish oil concentrate. Participants randomized to placebo took 3 placebo oil capsules per day, 2 in the morning with a meal and 1 in the afternoon with a meal and 1 placebo LA tablet in the morning with a meal. Fish oil concentrate and soybean oil placebo capsules were supplied by Nordic Naturals, Watsonville, CA and the LA and LA placebo tablets were supplied by Meda Pharma, Bad Homburg, Germany.

\section{Omega-3 and LA measures}

$\omega-3$, DHA, and EPA, were measured in red blood cell (RBC) membranes using methods described by Ruyle et al. [12] at the Oregon Health \& Science University (OHSU) Lipid Lab. RBC fatty acids are good indicators for tissue fatty acid levels. EPA and DHA levels are indicated by percent of total fatty acids measured in RBC membranes. A higher percent reflects higher amounts of DHA and EPA incorporated into membranes. Change from baseline at 6 months and 12 months was compared between groups. Because LA's clearance is rapid [13], LA was measured at one in-clinic visit, 45 and 90 minutes post oral dosing. Subjects took their usual study medications in clinic (one LA capsule plus two fish oil capsules or corresponding placebo capsules), blood was drawn 45 and 90 minute post-oral administration. Plasma samples were stored at $-70^{\circ} \mathrm{C}$ and measured in batches at OHSU. LA levels were determined by liquid chromatography tandem mass spectrometry (LC/MS/MS) using an adaptation of the method of Chen et al. in a 96 well plate format [14]. Levels are expressed in $\mu \mathrm{g} / \mathrm{ml}$, the 45 and 90 minute time point levels were averaged for a single mean serum LA level.

\section{F2-isoprostane levels}

Urine F2-isoprostane measurements were used to avoid ex vivo lipid peroxidation that can occur with plasma samples and to give a measure that reflects systemic oxidative damage over time $[15,16]$. Levels were standardized by reporting as $\mathrm{ng} / \mathrm{mg}$ creatinine. Clean catch urine was collected for each subject and a $1 \mathrm{ml}$ aliquot was immediately frozen on dry ice; all samples were kept frozen at $-70^{\circ} \mathrm{C}$ until analyzed. F2-isoprostanes in urine were quantified using gas chromatography with negative ion chemical ionization mass spectrometry and selective ion monitoring [15, 16]. Samples were analyzed in batches at the University of Washington, Seattle, WA.

\section{Cognitive and functional measures}

MMSE is a measure of global cognitive function, and scores range from 0-30, with a lower score indicates greater cognitive impairment [17]. The ADAS-cog assesses general cognitive function over multiple domains [18]. A higher score indicates greater impairment and the range of scores is 0 to 70. A single rater trained at OHSU's Oregon Alzheimer's Disease Center administered MMSE and ADAS-cog measures. A modified Alzheimer's Disease Cooperative Study ADL (ADCS ADL) was used to measure ADL/IADL [19]. The participant's caregiver/study partner rates basic and instrumental ADL. A higher ADL/IADL score indicates a worsening in functionality; scores range from 0 to 27 for ADL and 0 to 14 for IADL.

\section{Compliance and blinding}

Compliance was assessed by pill count at 6 months and 12 months. The study assessed the maintenance of blinding over 12 months by asking the participant's study partner, the participant, and all research staff involved in administering outcome measures about knowledge of group assignment at 12 months.

\section{Safety measures}

Safety was evaluated by adverse events reported by the participant and their study partner, laboratory tests 


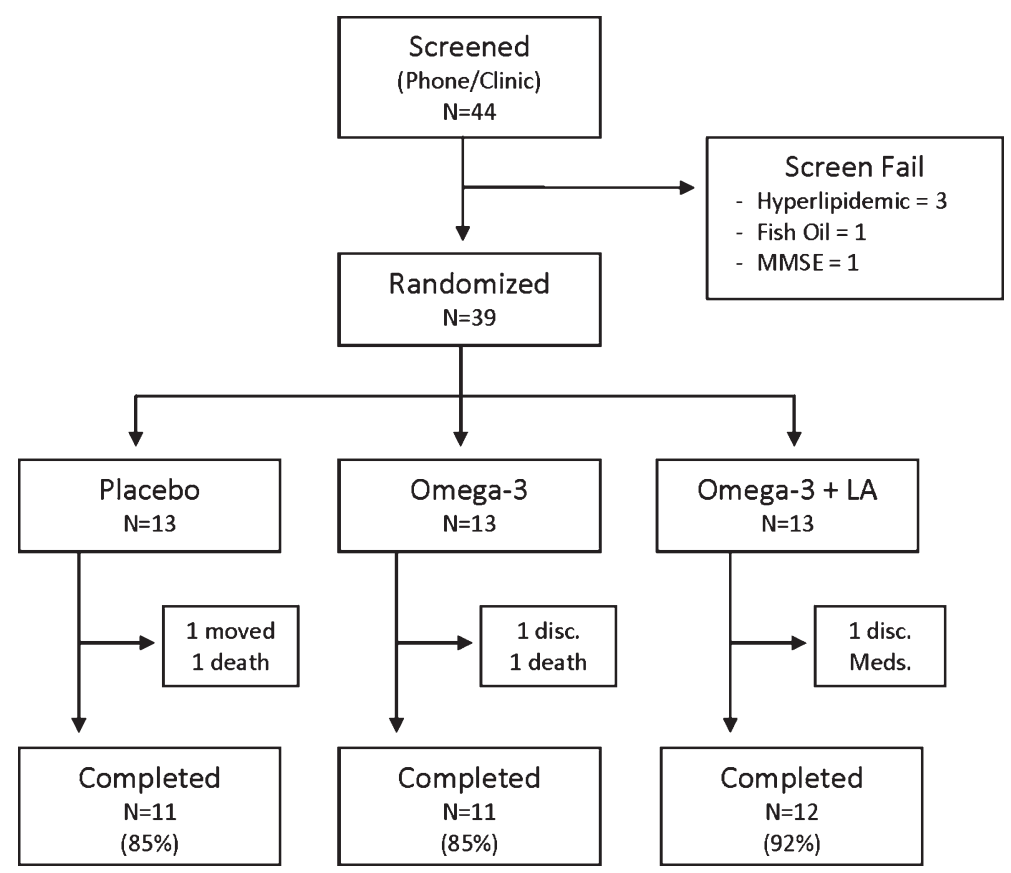

Fig. 1. Study flow.

(comprehensive metabolic panel and prothrombin time (PT/INR)), vital signs, and physical and neurological examinations.

\section{Statistical analysis}

Baseline characteristics were compared among placebo, $\omega-3$, and $\omega-3+$ LA, groups using Pearson chisquare or Fisher's exact tests for categorical variables and analysis of variance (ANOVA) for continuous variables. The primary outcome measure was the difference at 12 months in urine F2-isoprostane level adjusted for creatinine levels. Secondary outcome measures included MMSE, ADAS-cog, ADL, and IADL, and evaluated change in scores over 12 months. Because of significant group differences at baseline for F2-isoprostane levels between the $\omega-3$ group and the other two groups (placebo and $\omega-3+\mathrm{LA}), \mathrm{F} 2-$ isoprosatane level between groups was analyzed at 6 and 12 months using linear regression, rather than using linear mixed effects model that would measure repeated observations over time. For each time point, 6 months or 12 months, F2-isoprostane level was used as the dependent variable; independent variables included treatment group, age, and body mass index. For secondary outcome measures (ADAS-Cog, MMSE, ADL, and IADL), a linear mixed effects model was used. This method allows for correlation between repeated observations on each subject and provides valid inference in the presence of missing data as long as the data is missing at random (MAR). Each outcome was set as the dependent variable, and independent variables included treatment group, education level $(1=$ bachelor's degree or higher, $0=$ less than a bachelor's degree), and age. The group difference in slope was assessed by the interaction of intervention time by $\omega-3$ group, and intervention time by $\omega-3+$ LA, using the placebo group as the reference. Fischer's LSD was used to evaluate pairwise comparisons of means between placebo versus $\omega-3$ and placebo versus $\omega$ $3+$ LA. Bonferroni adjustment was used to account for multiple comparisons by dividing $p=0.05$ by five outcomes (F2-isoprostane, MMSE, ADAS-cog, ADL, IADL); significance was set at $p \leq 0.01$.

\section{RESULTS}

\section{Patient characteristics}

The CONSORT (Consolidated Standards of Reporting Trials) flowchart for the study is presented in Fig. 1. A total of 39 patients were randomized, 13 to each group. Except for F2-isoprostanes, there were no significant differences between groups in baseline characteristics (Table 1). 
Table 1

Baseline characteristics

\begin{tabular}{|c|c|c|c|c|}
\hline & $\begin{array}{c}\text { Placebo } \\
\text { Mean (SEM) }\end{array}$ & $\begin{array}{c}\omega-3 \\
\text { Mean (SEM) }\end{array}$ & $\begin{array}{c}\omega-3+\text { LA } \\
\text { Mean }(\text { SEM) }\end{array}$ & $p$ value \\
\hline Age (years) & $75.2(10.8)$ & $75.9(8.1)$ & $76.7(10.6)$ & $p=0.93$ \\
\hline Female & $54 \%$ & $39 \%$ & $39 \%$ & $p=0.74$ \\
\hline White & $100 \%$ & $100 \%$ & $100 \%$ & $p=1.00$ \\
\hline College or greater & $54 \%$ & $39 \%$ & $39 \%$ & $p=0.66$ \\
\hline Body mass index & $23.8(3.1)$ & $26.2(4.5)$ & $25.4(3.6)$ & $p=0.26$ \\
\hline MMSE & $22.2(3.1)$ & $20.7(2.7)$ & $22.5(3.0)$ & $p=0.27$ \\
\hline F2-isoprostane (ng/mg creatinine) & $1.5(0.2)$ & $3.6(1.0)$ & $1.4(0.2)$ & $p=0.03$ \\
\hline ADL & $3.3(1.0)$ & $2.2(.0 .3)$ & $1.5(0.6)$ & $p=0.23$ \\
\hline IADL & $10.0(1.8)$ & $10.8(1.1)$ & $6.8(1.9)$ & $p=0.13$ \\
\hline ADAS-Cog & $32.2(9.5)$ & $31.8(9.4)$ & $29.0(7.1)$ & $p=0.60$ \\
\hline *DHA & $4.4(1.0)$ & $5.1(1.3)$ & $5.0(1.8)$ & $p=0.39$ \\
\hline$* \mathrm{EPA}$ & $0.55(0.14)$ & $0.60(0.23)$ & $0.66(0.38)$ & $p=0.60$ \\
\hline$* *$ AD med use & $77 \%$ & $92 \%$ & $77 \%$ & $p=0.50$ \\
\hline
\end{tabular}

SEM is standard error of the mean. *Percent of total in red blood cell membranes. **AD med use indicates cholinesterase inhibitor or memantine.

Table 2

Percent fatty of total fatty acids in red blood cell membranes

\begin{tabular}{lcccc}
\hline & $\begin{array}{c}\text { Baseline } \\
(\text { mean, SEM) }\end{array}$ & $\begin{array}{c}\text { 6 Months } \\
(\text { mean, SEM) }\end{array}$ & $\begin{array}{c}\text { 12 Months } \\
(\text { mean, SEM) }\end{array}$ & $* p$ value \\
\hline DHA & & & & \\
Placebo & $4.4(0.3)$ & $4.0(0.3)$ & $4.1(0.3)$ & 0.41 \\
$\omega$-3 & $5.1(0.4)$ & $8.0(0.4)$ & $7.7(0.2)$ & $<0.001$ \\
$\omega$-3 + LA & $5.0(0.5)$ & $7.2(0.5)$ & $7.2(0.3)$ & 0.001 \\
EPA & & & & \\
Placebo & $0.6(0)$ & $0.6(0)$ & $0.6(0)$ & 0.95 \\
$\omega$-3 & $0.6(0.1)$ & $2.9(0.2)$ & $3.0(0.2)$ & $<0.001$ \\
$\omega$-3 + LA & $0.7(0.1)$ & $2.8(0.2)$ & $2.7(0.2)$ & $<0.001$ \\
\hline
\end{tabular}

SEM is standard error of the mean. ${ }^{*}$ Change from baseline to 12 months.

\section{Omega-3 levels, LA levels, blinding}

At 6 and 12 months, the groups that received fish oil showed a significant increase from baseline in the percent of DHA and EPA in red blood cell membranes (Table 2). Mean LA levels that were measured once in-clinic were: placebo, $0.03( \pm 0.03) \mu \mathrm{g} / \mathrm{ml} ; \omega$ $3,0.29( \pm 0.29) \mu \mathrm{g} / \mathrm{ml}$; and $\omega-3+\mathrm{LA}, 2.17( \pm 0.60)$ $\mu \mathrm{g} / \mathrm{ml}$. There was no significant difference between placebo and $\omega-3$ groups $(p=0.60)$; the $\omega-3+$ LA group had higher serum LA levels when compared to the placebo group $(p=0.002)$ and compared to the $\omega-3$ group ( $p=0.006)$. When asked about treatment assignment at the end of the study, the majority reported no knowledge of treatment assignment: research staff (100\%), AD participant (84\%), participant study partner $(81 \%)$.

\section{Urine F2-isoprostane levels}

Because the $\omega$ - 3 group had significantly higher baseline F2-isoprostane levels than the other two groups

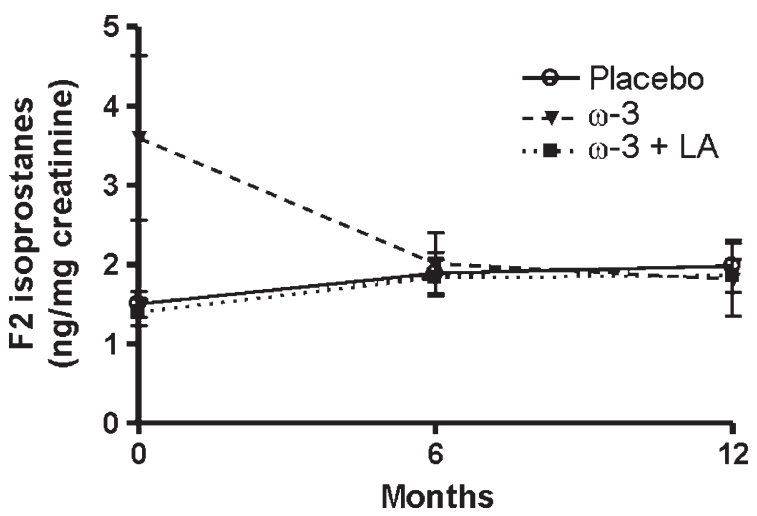

Fig. 2. F2 isoprostane levels. Standardized urine F2-isoprostane levels by adjusting for creatinine. Comparison between groups measured at baseline $(p=0.03), 6$ months $(p=0.78)$, and 12 months $(p=0.83)$ by linear regression adjusting for age and body mass index. Error bars indicate standard error of the mean.

$(p=0.03)$, it was difficult to interpret treatment effects on the change in F2-isoprostane levels after 12 months. There was no difference between groups on F2isoprostane levels at 6 months $(p=0.78)$ and 12 months $(p=0.83)$; concentrations at both time points were similar (Fig. 2).

\section{Cognitive measures (ADAS-cog, MMSE)}

The mean change in ADAS-cog over 12 months for the placebo group was 3.2 points $( \pm 2.1)$; for the $\omega-3$ group 4.4 points $( \pm 2.2)$, and for the $\omega-3+$ LA group 2.8 points $( \pm 2.0)$. The linear mixed effects model adjusting for age and education showed no difference in ADAS-cog between placebo and $\omega-3(p=0.86)$ or between placebo and $\omega-3+\mathrm{LA}(p=0.98)$. The mean 


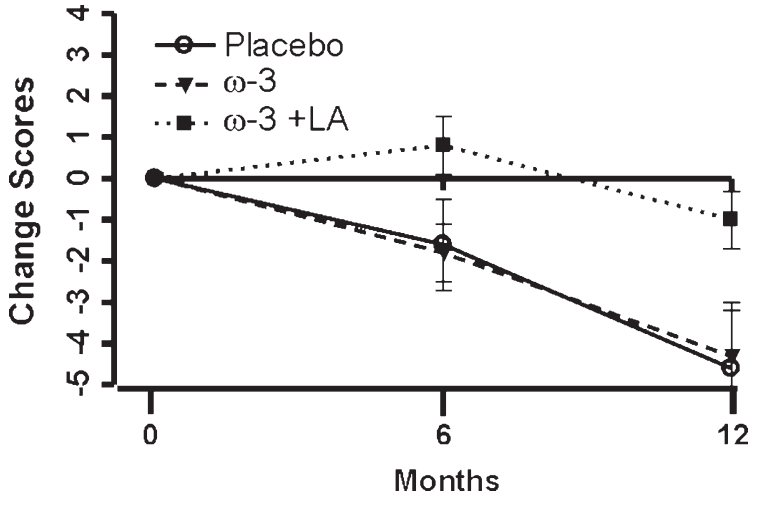

Fig. 3. Mini-Mental State Exam. Comparison of change scores for activities of daily living, using linear mixed effects model adjusting for age and education, error bars indicate standard error of the mean. A lower score reflects worsening of MMSE. No difference between placebo and $\omega-3$ was found over 12 months $(p=0.80)$. A significant difference was found between placebo and $\omega-3+$ LA over 12 months $(p<0.01)$.

change in MMSE over 12 months for the placebo group was -4.6 points $( \pm 1.4)$, for the $\omega-3$ group -4.3 points $( \pm 1.3)$, and for the $\omega-3+$ LA group -1.0 points $( \pm 0.7)$. In the linear mixed effects model adjusted for age and education level, there was no difference between placebo and $\omega-3(p=0.80)$, but there was a significant difference between placebo and $\omega-3+\mathrm{LA}(p<0.01)$, suggesting that the combination therapy decreased the rate of decline in MMSE over 12 months (Fig. 3).

\section{Functional measures (ADL/IADL)}

The mean change in IADL over 12 months for the placebo group was 4.2 points $( \pm 0.9)$, for the $\omega$-3 group 0.7 points $( \pm 1.0)$, and for the $\omega-3+$ LA group 0.9 points $( \pm 1.1)$. Linear mixed effects model adjusting for age and education level showed that compared to placebo both $\omega-3$ and $\omega-3+$ LA showed less decline in IADL $(p<0.01, p=0.01)$ (Fig. 4). The mean change in $\mathrm{ADL}$ over 12 months for the placebo group was 2.9 points $( \pm 0.7)$, for the $\omega$-3 group 2.5 points $( \pm 1.0)$, and for the $\omega-3+$ LA group 1.3 points $( \pm 0.8)$. Linear mixed effects model adjusting for age and education level showed no difference between placebo and $\omega$ $3(p=0.82)$ and no difference between placebo and $\omega-3+\operatorname{LA}(p=0.15)$.

\section{Safety}

The study treatments were well tolerated and most adverse events were mild. The most commonly

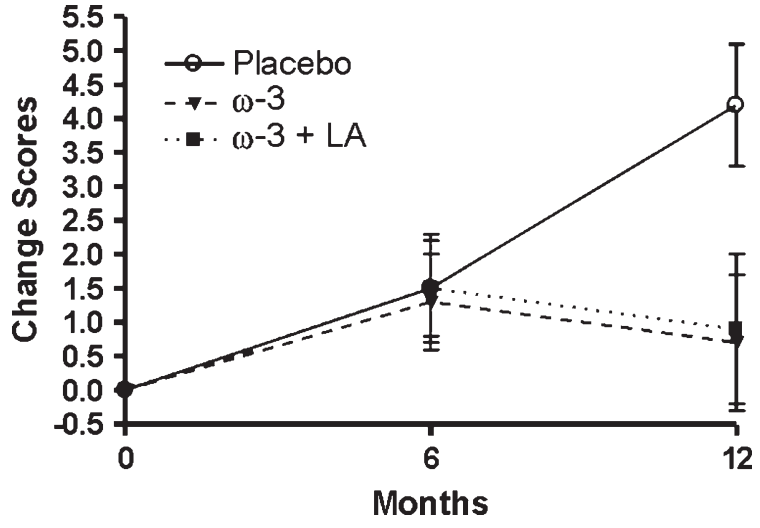

Fig. 4. Instrumental activities of daily living. Comparison of change scores for instrumental activities of daily living, using linear mixed effects model adjusting for age and education. Error bars indicate standard error of the mean. A higher score reflects worsening of IADL. A significant difference was found between placebo and $\omega-3$ and between placebo and $\omega-3+$ LA over 12 months $(p<0.01$, $p=0.01)$.

reported adverse events included the following: cold or flu (3 placebo, $2 \omega-3,2 \omega-3+\mathrm{LA}$ ), loose stools (3 placebo, $3 \omega-3+\mathrm{LA}$ ), dizziness ( 1 placebo, $2 \omega$ 3, $2 \omega-3+\mathrm{LA}$ ), falls (2 placebo, $1 \omega-3$ ). Two serious adverse events (SAE) occurred. One death occurred in the placebo group from complications after a urinary tract infection. One death occurred in the $\omega-3$ group from cardiac arrest. Neither SAE were deemed related to study medications. Mean values for the comprehensive metabolic panel and PT/INR were within normal limits at baseline and at 12 months (data not shown).

\section{DISCUSSION}

Because oxidative stress has been implicated in AD progression [20,21], the pilot study was designed to examine the effects of $\omega-3$ and $\omega-3+$ LA on peripheral F2-isoprostane levels. Compared to placebo, there was no significant difference at 6 or 12 months in the primary outcome measure, urinary $\mathrm{F} 2$ isoprostane levels. Additional post-hoc analysis of peripheral protein carbonyls (markers of protein oxidation) showed no baseline difference between groups and no change in levels over 12 months ( $p>0.20$, data not shown). Thus, there appeared to be no treatment effect on biomarkers of lipid or protein oxidation. For secondary measures of cognitive and functional impairment, the study did not demonstrate a treatment effect on ADAScog change over 12 months. However, both treatment 
arms showed a delay in progression of functional impairment (IADL) when compared to placebo over 12 months. In addition, the $\omega-3+$ LA group showed a slowing of global cognitive decline as measured by the MMSE that was not seen in the placebo or $\omega-3$ groups.

The positive effects observed in the combination therapy group on cognitive and functional measures is not associated with a decrease in biomarkers of oxidative stress, therefore the mechanism of action remains unclear. There is one reported study in $\mathrm{AD}$ evaluating the effects of supplementation with a combination of antioxidanst on cerebrospinal fluid (CSF) biomarkers; cognitive and functional measures. Subjects were randomized to one of three groups: 1) placebo, 2) antioxidant combination (daily dose, 800 IU $\alpha$-tocopherol, $500 \mathrm{mg}$ vitamin $\mathrm{C}, 900 \mathrm{mg}$ alpha lipoic acid), 3) Coenzyme Q10 (400 mg three times a day) [22]. Although the antioxidant combination group showed a reduction in CSF F2-isoprostane levels, this group also exhibited faster cognitive decline (MMSE) over 16 weeks than the placebo group. The authors concluded that although antioxidant effects had been achieved with the specific dose and combination of antioxidants, the rapid decline in MMSE scores raised "a caution" and a longer-term clinical trial of this antioxidant combination was warranted. In a transgenic model of cerebral amyloidosis ( $\mathrm{Tg} 2576)$, $\mathrm{Tg} 2576$ mice and wild type mice were fed a LA diet (1\%) or a control diet for 6 months then assessed for memory, oxidative stress (F2-isoprostanes, neuroprostanes), and amyloid- $\beta$ levels. LA-treated Tg2576 mice had significant improvement in learning and memory retention (Morris water maze task) compared to untreated mice. Although LA significantly reduced hipppocampal-dependent memory deficits, markers of oxidative stress (F2-isoprostanes and neuroprostanes) and amyloid- $\beta$ levels were not affected by LA treatment [23]. It is interesting to note that the pilot study outcomes from the $\omega$-3 + LA group mirror the Tg2576 animal study's finding, where improvements in cognitive measures were observed without improvements in oxidative stress measures. Furthermore, supplementation of an antioxidant combination in AD subjects was able to improve brain oxidative stress without improvement in cognition or daily function [22]. In agreement with the cited studies, the results from our pilot study do not support a positive association between antioxidant biomarkers and cognitive/functional outcomes. The combination of $\omega-3$ with LA may impact multiple mechanisms associated with $\mathrm{AD}$ pathogenesis that are not based on antioxidant capacity and these mech- anisms should be explored in future studies. Data from AD animal models support the ability of DHA to protect from synaptic and dendritic loss [24]. Oral LA has been reported in two open-label AD studies to delay cognitive decline and, in non-AD cohorts, has a positive effect on insulin resistance [25-27]. In multiple sclerosis studies, oral LA given to multiple sclerosis participants has been shown to increase cAMP production and can decrease immune cell secreted inflammatory cytokines by modulating cAMP [28, 29]. $\omega-3$ and LA have been shown to decrease s-ICAM-1 levels and therefore, may have an impact on inflammatory markers of vascular risk [30, 31]. The pilot study was not powered to assess whether outcomes observed were associated with biomarkers other than F2-isoprostane levels. A larger study is currently under way that will assess the effects of $\omega-3+$ LA on slowing the rate of cognitive and functional decline in $\mathrm{AD}$ (NCT01058941; ClinicalTrials.gov). The larger study is also designed to evaluate the combination therapy on peripheral biomarkers (IL-6, TNF- $\alpha$, lipid panel, insulin resistance) and brain changes (MRI total brain atrophy and white matter hyperintensity) that may be associated with AD progression.

The two largest placebo controlled studies evaluating $\omega-3$ in $\mathrm{AD}$ found no effect in slowing rate of decline in ADAS-cog, MMSE, or ADL [32, 33]. In agreement with the two larger studies, our pilot study showed no effect of $\omega-3$ in slowing rate of decline in ADAS-cog or MMSE, although $\omega-3$ did slow IADL decline when compared to placebo. In a review of EPA and DHA in atherosclerosis factors, the authors note that variability in dose, dose duration, and DHA to EPA ratio can influence reported outcomes [34]. There is no consensus in AD studies for evaluating a standard ratio or dose of DHA and EPA: Quinn et al. evaluated DHA at 2 grams/day; Freund-Levi et al. evaluated fish oil (DHA $1.7 \mathrm{~g} /$ day and EPA $0.6 \mathrm{~g} /$ day). This pilot study evaluated $\omega$-3 using 2-fold lower daily doses of DHA and a much lower ratio of DHA:EPA than the Quinn and Freund-Levi studies. As it is still unclear what ratio and dose of DHA and EPA would be beneficial in AD, it may be that the observed slowing in IADL progression in the $\omega-3$ only group reflects a beneficial dose and ratio that constitutes a higher EPA to DHA ratio and a lower daily dose of DHA.

There are two published open-label studies evaluating LA in $\mathrm{AD}$ patients, both from the same group in Germany [25, 26]. The first study followed nine patients with $\mathrm{AD}$ and related dementias for approximately 1 year after treatment with acetylcholinesterase inhibitors and $600 \mathrm{mg} /$ day of LA and reported a 
stabilization of cognitive function measured by MMSE and ADAS-cog after one year [25]. The investigators extended the study to 43 AD patients over an observation period of 48 months, in a subset of mildly impaired patients (ADAS-cog score <15); they report a yearly change in MMSE of -0.6 points and yearly change in ADAS-cog at+1.2 points, which is significantly less decline than reported for these measures in mildly impaired AD patients that did not receive LA [26]. The study suggested a potential benefit of LA therapy in mildly impaired AD patients. Our pilot study found that $\omega-3+$ LA resulted in less decline of MMSE when compared to placebo or $\omega-3$ alone, although we do not know if the effects observed in the $\omega-3+\mathrm{LA}$ group were a result of combining the two dietary supplements or from LA alone as we did not evaluate a LA only group.

It is possible that there is added protective benefit in combining an antioxidant like LA to $\omega-3$ as in a related earlier study, higher baseline levels of carotenoids (antioxidants) and DHA were associated with higher MMSE scores [35]. In a cross-sectional study of cognitively intact elderly, high plasma levels of EPA and DHA were associated with low white matter hyperintensity burden and higher executive function performance. In addition higher plasma levels of an "antioxidant" cluster (vitamins C, E, B, and D) was associated with greater total brain volume and higher scores on global cognitive function [36]. The two studies support the idea that adding antioxidants to $\omega-3$ may enhance benefit in protecting cognitive function. The cross-sectional study in cognitively intact elderly suggests that EPA/DHA and antioxidants have different and specific mechanisms of action on preserving brain integrity and that combining nutrients may have more favorable outcomes than using a single nutrient or combining nutrients with the same mechanism of action.

There are a number of factors that could have impacted the treatment effects we observed. The study had a small sample size with multiple outcomes that increase our chance of finding a false positive outcome. When we use Bonferronni adjustment for multiple outcomes, setting significance at $p \leq 0.01$, our main findings favoring the combination treatment in MMSE and IADL remained significant $(p<0.01, p=0.01)$. The $\omega-3$ only group also remains significant for delaying IADL progression $(p<0.01)$. Most, but not all, of the participants were taking a cholinesterase inhibitor or a NMDA-receptor antagonist (memantine) and outcomes could have been influenced by the use of these medications. In a mixed effects model analysis, we assessed cholinesterase inhibitor and NMDA-receptor agonist use (individually) on each outcome (MMSE, ADAS-cog, ADL, IADL) and did not see a significant effect on any outcome ( $p$-values $>0.30$, data not shown). The $\omega$ - $3+$ LA group showed less decline on MMSE and IADL, but we did not find a corresponding effect in ADAS-cog and ADL. Differential completer numbers at 12 months could account for this incongruity. All subjects who completed the study could perform the MMSE at 12 months while 10/13 (77\%) in placebo group and 12/13 (92\%) in $\omega-3+$ LA group were able to perform ADAS-cog. Although change in ADAS-cog between groups was not significant, a higher percentage in the combination group was able to do the ADAS-cog which is in the expected direction of MMSE findings if this were a factor. Mean baseline ADL scores were relatively low reflecting higher function on basic living skills such as bathing and eating but mean baseline IADL scores were in a moderate range. Higher function on ADL at baseline may have blunted the ability to distinguish a change between groups over 12 months. Although not significant, mean ADL change scores for each group follow the general pattern of the IADL scores with the placebo group having greater decline than $\omega-3+\mathrm{LA}$ and $\omega-3$ groups. As IADL measures more complex tasks such as using the telephone or preparing meals, it may have been more sensitive to $\mathrm{AD}$ progression over time than the more basic ADL measures.

\section{CONCLUSIONS}

In a small pilot study combining $\omega-3$ with LA slowed both cognitive and functional decline in mild to moderately impaired AD participants over 12 months, and the combination appears to be safe at the doses evaluated. Because the results are derived from a small sample size, caution in broadly interpreting the outcomes reported is warranted. A larger pilot trial is underway to further assess the benefit and potential mechanism of action of this novel combination for AD.

\section{ACKNOWLEDGMENTS}

The research was supported by the National Institutes of Health/National Institute of Aging (NIH/NIA) R21AG023805, NIH/NIA AG08017, and NIH General Clinical Research Grant M01RR00334. Nordic Natural, Watsonville, CA, USA, supplied the fish oil and placebo oil, and Meda Pharma, Bad Homburg, 
Germany, supplied the lipoic acid and placebo. We would like to thank Carl Cotman and Wycliffe Opii at the Institute for Brain Aging \& Dementia, University of California Irvine, for measuring plasma protein carbonyl levels.

Authors' disclosures available online (http://www.jalz.com/disclosures/view.php?id=1844).

\section{REFERENCES}

[1] Cummings JL, Cole G (2002) Alzheimer's disease. JAMA 287, 2335-2338.

[2] Fetterman JW, Zdanowicz MM (2009) Therapeutic potential of n-3 polyunsaturated fatty acids in disease. Am J Health Sys 66, 1169-1179.

[3] Jicha GA, Markesbery WR (2010) Omega-3 fatty acids: Potential role in the management of early Alzheimer's disease. Clin Invest Aging 5, 45-61.

[4] Anderson BM, Ma DW (2009) Are all n-3 polyunsaturated fatty acids created equal? Lipid Health Dis $\mathbf{8}, 33$.

[5] Barberger-Gateau P, Raffaitin C, Letenneur L, Berr C, Tzourio C, Dartigues JF, Alpérovitch A (2007) Dietary patterns and risk of dementia: The Three-City cohort study. Neurology 69 1921-1930.

[6] Kalmijn S, Launer LJ, Ott A, Witteman JC, Hofman A, Breteler MM (1997) Dietary fat intake and the risk of incident dementia in the Rotterdam Study. Ann Neurol 42, 776-782.

[7] Morris MC, Evans DA, Bienias JL, Tangney CC, Bennett DA, Wilson RS, Aggarwal N, Schneider J (2003) Consumption of fish and n-3 fatty acids and risk of incident Alzheimer disease. Arch Neurol 60, 940-946

[8] Shay KP, Moreau RF, Smith EJ, Smith AR, Hagen TM (2009) Alpha-lipoic acid as a dietary supplement: Molecular mechanisms and therapeutic benefit. Biochim Biophys Acta 10, 1149-1160.

[9] Packer L, Roy S, Sen CK (1997) Alpha-lipoic acid: A metabolic antioxidant and potential redox modulator of transcription. Adv Pharmacol 38, 79-101.

[10] Lee HA, Hughes DA (2002) Alpha-lipoic acid modulates NF-kappaB activity in human monocytic cells by direct interaction with DNA. Exp Gerontol 37, 401-410.

[11] Helmersson J, Larsson A, Vessby B, Basu S (2005) Active smoking and a history of smoking are associated with enhances prostaglandin F (2 alpha), interleukin 6 and F2 isoprostane formation in elderly men. Atheroslcerosis $\mathbf{1 8 1}$ 201-207.

[12] Ruyle M, Connor WE, Anderson GJ, Lowensohn RI (1990) Placental transfer of essential fatty acids in humans: Venousarterial difference for docosahexaenoic acid in fetal umbilical erythrocytes. Proc Natl Acad Sci U S A 87, 7902-7906.

[13] Biewenga GP, Haenen GR, Bast A (1997) The pharmacology of the antioxidant lipoic acid. Gen Pharmacol 29, 315-331.

[14] Chen J, Jiang W, Cai J, Tao W, Gao X, Jiang X (2005) Quantification of lipoic acid in plasma by high-performance liquid chromatography-electrospray ionization mass spectrometry. $J$ Chromatogr B Analyt Technol Biomed Life Sci 824, 249-257.

[15] Mori TA, Croft KD, Puddey IB, Beilin LJ (1999) An improved method for the measurement of urinary and plasma F2isoprostanes using gas chromatography-mass spectrometry. Anal Biochem 268, 117-125.

[16] Helmersson J, Basu S (2001) F(2)-isoprostane and prostaglandin $\mathrm{F}(2$ alpha) metabolite excretion rate and day to day variation in healthy humans. Prostaglandins Leukot Essent Fatty Acids 65, 99-102.

[17] Folstein M, Folstein SE, McHugh PR (1975) "Mini-mental state". A practical method for grading the cognitive state of patients for the clinician. J Psychiatr Res 12, 189-198.

[18] Rosen WG, Mohs RC, Davis KL (1984) A new rating scale for Alzheimer's disease. Am J Psychiatry 141, 1356-1364.

[19] Galasko D, Bennett D, Sano M, Ernesto C, Thomas R, Grundman M, Ferris S (1997) An inventory to assess activities of daily living for clinical trials in Alzheimer's disease. The Alzheimer's Disease Cooperative Study. Alzheimer Dis Assoc Disord 11(S2), S33-S39.

[20] Grundman M, Delaney P (2002) Antioxidant strategies for Alzheimer's disease. Proc Nutr Soc 61, 191-202.

[21] Pratico D, MY Lee V, Trojanowski JQ, Rokach J, Fitzgerald GA (1998) Increased F2-isoprostanes in Alzheimer's disease: Evidence for enhanced lipid peroxidation in vivo. FASEB J12, 1777-1783.

[22] Galasko DR, Peskind E, Clark CM, Quinn JF, Ringman JM, Jicha GA, Cotman C, Cotttrell B, Montine TJ, Thomas RG, Aisen P (2012) Antioxidants for Alzheimer's disease: A randomized clinical trial with cerebrospinal fluid biomarker measures. Arch Neurol 69, 836-841.

[23] Quinn JF, Bussiere JR, Hammond RS, Montine TJ, Henson E, Jones RE, Stackman RW Jr (2007) Chronic dietary alphalipoic acid reduces deficits in hippocampal memory of aged Tg2576 mice. Neurobiol Aging 28, 213-225.

[24] Cole GM, Frautschy SA (2010) DHA may prevent age-related dementia. J Nutr 140, 869-874

[25] Hager K, Marahrens A, Kenklies M, Riederer P, Münch G (2001) Alpha-lipoic acid as a new treatment option for Azheimer type dementia. Arch Gerontol Geriatr 32, 275282.

[26] Hager K, Kenklies M, McAfoose J, Engel J, Münch G (2007) Alpha-lipoic acid as a new treatment option for Alzheimer's disease-a 48 months follow-up analysis. J Neural Transm Suppl 72, 189-193.

[27] Kamenova P (2006) Improvement of insulin sensitivity in patients with type 2 diabetes mellitus after oral administration of alpha-lipoic acid. Hormones 5, 251-258.

[28] Salinthone S, Yadav V, Schillace RV, Bourdette DN, Carr DS (2010) Lipoic acid attenuates inflammation via cAMP and protein kinase A signaling. PLoS One 5, e13058.

[29] Salinthone S, Schillace RV, Tsang C, Regan JW, Bourdette DN, Carr DW (2011) Lipoic acid stimulates cAMP production via $\mathrm{G}$ protein-coupled receptor-dependent and -independent mechanisms. J Nutr Biochem 22, 681-690.

[30] Yadav V, Marracci GH, Munar MY, Cherala G, Stuber LE, Alvarez L, Shinto L, Koop DR, Bourdette DN (2010) Pharmacokinetic study of lipoic acid in multiple sclerosis: Comparing mice and human pharmacokinetic parameters. Mult Scler 16, 387-397.

[31] Yang Y, Lu N, Chen D, Meng L, Zheng Y, Hui R (2012) Effects of n-3 PUFA supplementation on plasma soluble adhesion molecules: A meta-analysis of randomized controlled trials. Am J Clin Nutr 95, 972-980.

[32] Quinn JF, Raman R, Thomas RG, Yurko-Mauro K, Nelson EB, Van Dyck C, Galvin JE, Emond J, Jack CR Jr, Weiner M, Shinto L, Aisen PS (2010) Docosahexaenoic acid supplementation and cognitive decline in Alzheimer disease. JAMA 304, 1903-1911.

[33] Freund-Levi Y, Eriksdotter-Jönhagen M, Cederholm T, Basun H, Faxén-Irving G, Garlind A, Vedin I, Vessby B, Wahlund LO, Palmblad J (2006) Omega-3 fatty acid treatment in 174 patients with mild to moderate Alzheimer disease: OmegaAD 
study: A randomized double-blind trial. Arch Neurol 10, 14021408.

[34] Kelley DS, Adkins Y (2012) Similarities and differences between the effects of EPA and DHA on markers of atherosclerosis in humans subjects. Proc Nutr Soc 71, 322331.

[35] Wang W, Shinto L, Connor WE, Quinn JF (2008) Nutritional biomarkers in Alzheimer's disease: The association between carotenoids, n-3 fatty acids, and dementia severity. J Alzheimers Dis 13, 31-38.

[36] Bowman GL, Silbert LC, Howieson D, Dodge HH, Traber MG, Frei B, Kaye JA, Shannon J, Quinn JF (2012) Nutrient biomarker patterns, cognitive function, and MRI measures of brain aging. Neurology 78, 241-249. 